Verkehrsplanung auf Stadtteilebene

\title{
Mobilität in klimaneutralen Stadtquartieren - elektrisch, multimodal und vernetzt
}

\author{
Lärm, Abgase und Unfallgefahr sind neben \\ der Flächendominanz parkender Pkw zentrale \\ Probleme für die derzeitige Stadt- und Verkehrs- \\ entwicklung. Das Projekt „City2Share“ gibt \\ Anhaltspunkte für ökologische Alternativen. \\ Von Uta Bauer, Thomas Stein und Victoria Langer
}

O bwohl für ein Fünftel der klimaschädlichen Treibhaugase verantwortlich ist der Verkehrssektor bislang seinen Beitrag zu den Klimaschutzzielen weitgehend schuldig geblieben. Während der Treibhausgas-Ausstoß in den vergangenen Jahren in anderen Bereichen gesunken ist, ist er im Verkehr im Jahr 2017 sogar gestiegen. Ein konsequentes Umsteuern auf allen Handlungsebenen ist deshalb dringend geboten. Den Kommunen kommt dabei als wichtiger lokaler Akteur bei der Steuerung und Organisation von Mobilitätsnachfragen und -bedürfnissen eine zentrale Rolle zu. Das vom Bundesministerium für Umwelt, Naturschutz und nukleare Sicherheit geförderte Projekt "City2Share“ (Laufzeit: 2016-2020) soll zeigen, ob und wie wohnungsnahe, geteilte Elektromobilitätsangebote und neue Lieferkonzepte einen wirksamen Beitrag auf dem Weg zu klimaneutralen Stadtquartieren leisten können. Insgesamt zehn Partner aus Verwaltung, Forschung und Industrie sind an der Erarbeitung von praxisnahen Lösungen beteiligt.

\section{Mobilitätsstationen als Ankerpunkte multimodaler Mobilität}

Welche Rolle kommt neuen Mobilitätskonzepten bei der Gestaltung klimaneutraler Stadtquartiere zu? Neben energieoptimiertem Bauen und der energetischen Sanierung des Gebäudebestandes gehört eine umweltverträgliche Mobilität zu den wichtigsten Handlungsfeldern (BMVBS 2012). Quartiere dienen meist als Quelle und Ziel individueller Wege. Daher gilt es, bereits hier Grundlagen zu schaffen, um Verkehr zu vermeiden oder auf emissionsfreie und ressourcenschonende Verkehrsmittel zu verlagern. Die Quartiersstruktur und die im unmittelbaren Wohnumfeld verfügbaren Mobilitätsoptionen haben dabei einen entscheidenden Einfluss auf die Verkehrsmittelwahl. Gerade in Städten sind viele Wege kürzer als $5 \mathrm{~km}$ und somit auch gut per Fahrrad oder zu Fuß zurückzulegen (BMVI 2018). Geteilte Mobilitätsangebote in Quartieren sollen ein multimodales Mobilitätsverhalten unterstützen und vor allem eine Alternative zu (Privat-)Pkw-Fahrten und Pkw-Besitz bieten (FIS 2013).

Wie solche Ansätze in Bestandsquartieren umgesetzt werden können, wird im Projekt "City2Share" erprobt. Ausgewählte Modellquartiere in Hamburg und München dienen als Reallabore für Maßnahmen, die auf emissionsarme Mobilität, effizientere Nutzung des Straßenraums und eine verbesserte Aufenthalts- und Wohnumfeldqualität abzielen. Ein Kernelement des Projekts sind die zwischen 2017 und 2019 gebauten Elektro-Mobilitätsstationen in den Münchner Innenstadtquartieren Isarvorstadt-Ludwigsvorstadt. Ziel dieser Stationen ist es, durch die vernetzte Mobilität und die Bereitstellung neuer Informations- und Zugangssysteme (multimodale Buchbarkeit über eine App bzw. Informationen zu multimodalen Echtzeitdaten über Stelen) den Umstieg vom Pkw auf umweltfreundlichere und platzsparendere Mobilitätsangebote zu erleichtern. Jede Mobilitätsstation zeichnet sich durch unterschiedliche Ausstattungsmerkmale (z. B. Bikesharing und Carsharing Angebote) und Anbindungsqualitäten durch räumliche Nähe zu U-Bahn Haltestellen oder Buslinien an den ÖPNV aus. Wie diese Angebote in den unterschiedlichen Konfigurationen genutzt werden und welche Auswirkungen auf Stadtraum, Umwelt und Mobilitätsverhalten der Anwohnerinnen und Anwohner zu beobachten sind, wird derzeit in der begleitenden Wirkungs- und Prozessanalyse untersucht.

Neben der Bereitstellung von geteilten Mobilitätsangeboten können Mobilitätsstationen auch ein potenzieller Gewinn für den öffentlichen Raum durch eine Platzaufwertung darstellen. So wurde im Projekt "City2Share“ der vormals durch parkende Autos zugestellte Zenettiplatz im Zuge des Baus der Mobilitätsstation in Kooperation mit der Landeshauptstadt München und verschiedenen Initiativen zur „Piazza Zenetti“ umgestaltet.

\section{Wirkungen von Mobilitätsstationen und -angeboten}

Positive Umweltwirkungen werden grundsätzlich durch Verkehrsverlagerung, -verminderung und -vermeidung erreicht. Für die Analyse der Umweltwirkungen werden im Laufe des Projektes fahrzeugspezifische und verhaltensspezifische Ein- 
sparungspotenziale betrachtet. Erste Erfahrungen aus Erhebungen (Passantenbefragungen, Fokusgruppen) in München zeigen, dass viele Anwohnende die innerstädtischen Räume durch den Kfz-Verkehr belastetet sehen und sich eine Stärkung anderer Mobilitätsformen wünschen. Der Ausbau von eher „klassischen“ Mobilitätsangeboten, wie dem ÖPNV oder der Verbesserung der Radverkehrsinfrastruktur, steht bei den Anwohnenden dabei an erster Stelle der gewünschten Verbesserungen. Neue Mobilitätsangebote wie Car- oder Bikesharing stoßen zwar auf grundsätzliches Interesse; genutzt werden sie jedoch nur sehr begrenzt. Diese Tendenz aus dem Münchener Reallabor lässt sich auch an den aktuellen Zahlen der Verkehrserhebung „Mobilität in Deutschland“ (BMVI 2018) ablesen. Weitere Erkenntnisse aus dem Untersuchungsgebiet von "City2Share“ zeigen, dass sich die Nutzergruppe von Free-floating Carsharing noch auf männliche Personen zwischen 30 und 39 Jahren konzentriert.

Auch für Pkw-Besitzer/innen scheint ein Carsharing-Angebot allein noch kein Grund für die Kfz-Abschaffung zu sein. Obwohl die Befragten ihr Auto nur selten nutzen, ist der eigene Wagen für sie eine wertvolle „Mobilitätsreserve“ für große Einkäufe und Ausflugsfahrten am Wochenende. Für diese Bedarfe wären eher Lastenräder oder stationsgebundenes Carsharing das geeignetere Angebot.

So bleiben grundsätzliche Fragen in der Ausgestaltung und Wirkung neuer Mobilitätsangebote in Verbindung mit Mobilitätsstationen offen. Welche Angebote führen tatsächlich dazu, dass die Kfz-Fahrleistung sinkt? Trägt Free-floating Carsharing $\mathrm{zu}$ einer besseren Umweltbilanz bei oder werden Wege, die vorher mit Bussen und Bahnen oder dem Fahrrad erledigt wurden, nun mit dem Pkw gefahren? Wie schafft man ein stationsgebundenes Pkw-Angebot, das für die Anbieter dennoch wirtschaftlich ist? Welche zusätzlichen Anreize und Rahmenbedingungen sind für eine Abkehr vom privaten Pkw nötig?

\section{Quartierslogistik mit Lastenrad}

Neben den Belastungen durch den motorisierten Individualverkehr führt auch der Lieferverkehr auf der „letzten Meile“ zu immer größeren Beeinträchtigungen in dicht bebauten Innenstadtquartieren (Prümm et al. 2017). Das starke und anhaltende Wachstum im Online-Handel schlägt sich in den Städten in mehr Lieferfahrzeugen nieder, die in zweiter Reihe, auf Gehwegen und in Kreuzungsbereichen parken und somit viele
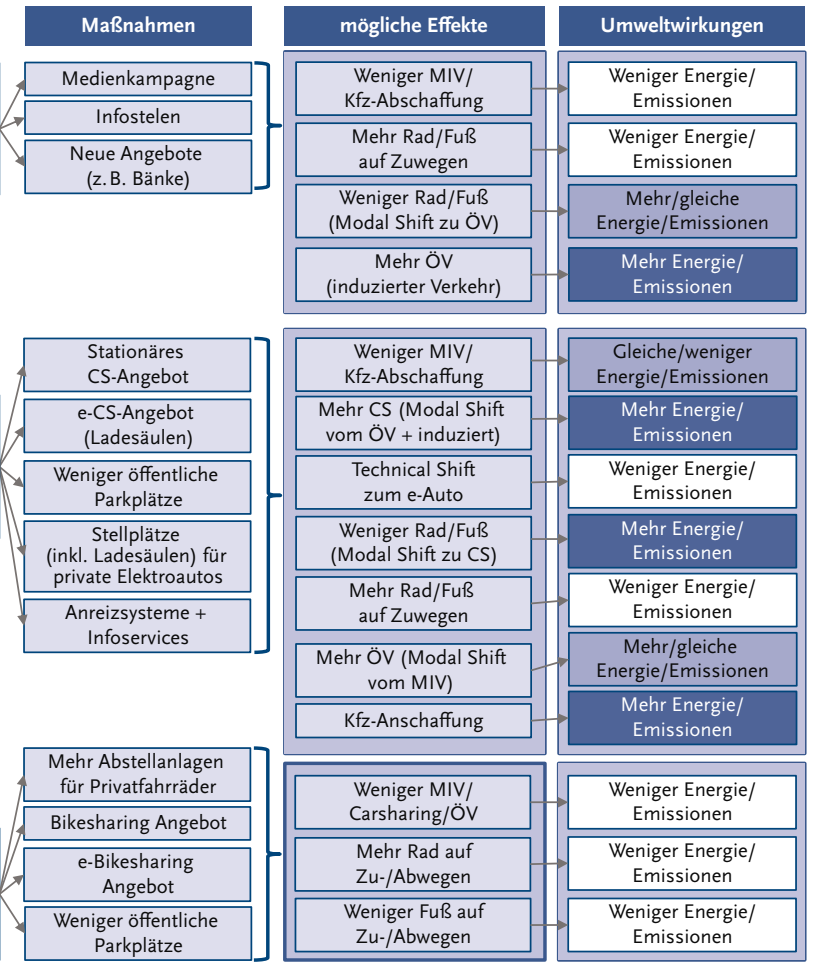

2Share mit möglichen Effekten

(Quelle: TU Dresden 2017, Professur für Verkehrsökologie)

Verkehrsteilnehmende gefährden. Nicht zu vernachlässigen ist, dass Lieferverkehre auch die bestehenden Flächenkonkurrenzen im öffentlichen Raum weiter verschärfen.

Um die „letzte Meile“ stadtverträglicher zu gestalten, wurden im Rahmen von „City2Share“ an drei Standorten im Sommer 2017 „Mikro-Depots“ errichtet. Zwei Container und ein Anhänger für die jeweiligen Modellquartiere bilden jeweils ein Feinverteilzentrum von dem aus der Projektpartner UPS, die Sendungen im Gebiet emissionsfrei, mit zwei- oder vierrädrigen Elektrofahrzeugen, zustellt. Ziel ist es, ein insgesamt übertragbares e-Lieferkonzept für innerstädtische Wohnquartiere zu entwickeln.

Die wissenschaftliche Begleitforschung zeigt, dass der erprobte Mikro-Depot-Ansatz eine sinnvolle Möglichkeit ist, Lieferverkehr in Innenstädten zu bündeln und stadtverträglicher abzuwickeln (Lindloff et. al 2018). Gleichzeitig erfährt das Projekt durch seine Transparenz und Sichtbarkeit bei den Anwohnenden eine hohe Akzeptanz. Nach eigenen Angaben hat UPS mit dem Aufbau der Mikro-Depots bereits 14 Dieselfahrzeuge in der bayrischen Hauptstadt eingespart und sieht Potenziale, das Konzept, auch nach Ende der Projektlaufzeit, auf das gesamte Stadtgebiet auszuweiten.

Aus Sicht von UPS können durch das neue Konzept, neben den ökologischen Einsparungen auch die Kosten für die Fahrzeugflotte erheblich reduziert werden, da (E-)Lastenräder in der Beschaffung und im Betrieb deutlich preisgünstiger sind als 


\section{„Der Verkehrssektor ist seinen Beitrag zu den Klimaschutzzielen bisher schuldig geblieben. Mobilitätsstationen in Quartieren können hier Abhilfe schaffen."}

konventionelle, motorbetriebene Lieferfahrzeuge. Außerdem ist für das Steuern eines (E-)Lastenrades kein Führerschein erforderlich, sodass vor dem Hintergrund des erheblichen Personalmangels in der Lieferbranche der Einsatz von (E-)Lastenrädern den Unternehmen bessere Chancen und mehr Flexibilität bei der Suche nach Mitarbeitenden geben kann.

Zentral für den Erfolg des neuen Lieferkonzeptes ist die Identifizierung, Sicherung und Bereitstellung der Flächen für die Depots. Geeignete Standorte und Flächen sollten daher bei Neu- und Umbaumaßnahmen in Quartieren vorgesehen und vor allem freigehalten werden. Aus technologischer Sicht bestehen künftige Aufgaben in der Förderung und Beschaffung emissionsarmer, elektrisch betriebener Fahrzeuge und im Ausbau einer öffentlichen Ladeinfrastruktur, um die Bedarfe zur erfolgreichen Inbetriebnahme von ökologischen Flotten im Lieferverkehr zu sichern.

\section{Erste Schlussfolgerungen}

Im Kontext von Klimaschutz, immer höherem Flächenverbrauch für den Kfz-Verkehr und (damit einhergehend) stetig wachsenden städtischen Verkehrsproblemen kommen geteilten Mobilitätsangeboten und einem anderen Umgang mit Lieferverkehren künftig eine besondere Bedeutung zu. Die ersten Erfahrungen aus „City2Share“ zeigen, dass neue Konzepte, wie Mikro-Konsolidierungszentren oder die Bündelung geteilter Mobilitätsangebote an Mobilitätsstationen, potenziell innerstädtische Quartiere von Verkehr entlasten können. Die Fragen, in welcher Konfiguration und Gestalt Mobilitätsstationen am ehesten einen Mobilitätswandel unterstützen und welche konkreten kommunalen Steuerungsinstrumente und Rahmensetzungen dafür nötig sind, sollen zum Ende des Projekts genauer beantwortet werden. Aus dem bisherigen Umsetzungsprozess in "City2Share" können jedoch erste wichtige Umsetzungsfaktoren identifiziert werden:

I Mobilitätsstationen und Paket-Depots sind ressourcen- und flächenschonend, benötigen jedoch ebenfalls Platz. Eine der Herausforderungen ist es, innerstädtische Standorte zu identifizieren und Flächen bereitzuhalten (bspw. im Zuge des Neu- oder Umbaus von Quartieren).
I Die Angebote sollten die spezifischen Mobilitätsbedarfe aufgreifen und neben positiven sozialen und wirtschaftlichen Effekten die Umwelt entlasten und somit einen nachweisbaren ökologischen Nutzen haben.

- Die Bevölkerung und insbesondere Anwohnende sollten von Anfang an in die Planungen eingebunden werden, um Akzeptanz für die Konzepte zu schaffen.

I Die eingesparten Flächen sollten in attraktivere Aufenthaltsflächen umgewidmet werden.

\section{Literatur}

BMVBS [Bundesministerium für Verkehr, Bau und Stadtentwicklung] (Hrsg.) (2012): EQ - Anforderungen an energieeffiziente und klimaneutrale Quartiere. ExWoSt-Informationen 42/2, Bonn, August 2012.

BMVI [Bundesministerium für Verkehr und digitale Infrastruktur] (Hrsg.) (2018): Mobilität in Deutschland - MiD. Ergebnisbericht, Bonn, Dezember 2018

FIS [Forschungsinformationssystem] (2013): Förderung des multi- und intermodalen Personenverkehrs. Im Internet unter: http://www.forschungsinformationssystem.de/servlet/is/354189.

Lindloff, K. et al. (2018): „Neue Konzepte des (E-) Lieferverkehrs in den Städten“. Ergebnisse aus dem Städtenetzwerk des Forschungsprojektes „City2Share“. Im Internet unter: http://www.city2share.de/info/ City2Share_Empfehlungspapier\%20E-Lieferverkehr_20180719.pdf.

Prümm, D. et al. (2017): Aufbruch auf der letzten Meile - Neue Wege für die städtische Logistik. Im Internet unter: https://www.pwc.de/de/transportund-logistik/pwc-studie-aufbruch-auf-der-letzten-meile.pdf.
AUTOR/INNEN + KONTAKT

Uta Bauer ist Projektleiterin am Deutschen Institut für Urbanistik. Tel.: +49 30 39001-151, E-Mail: bauer@difu.de

Thomas Stein ist wissenschaftlicher Mitarbeiter am Deutschen Institut für Urbanistik. Tel.: +49 30 39001-181, E-Mail: stein@difu.de

Victoria Langer ist wissenschaftliche Mitarbeiterin am Deutschen Institut für Urbanistik. Tel.: +49 30 39001-257, E-Mail: langer@difu.de
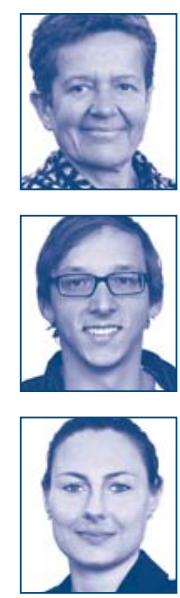